\title{
ANÁLISIS DE ASPECTOS QUE INCREMENTAN EL RIESGO DE INUNDACIONES EN COLOMBIA
}

\author{
KARIME SEDANO-CRUZ ${ }^{1}$, YESID CARVAJAL-ESCOBAR ${ }^{2}$, ÁLVARO \\ JAVIER ÁVILA DÍAZ
}

Recibido el 11 de febrero de 2013 y aprobado el 12 de abril de 2013

\section{RESUMEN}

Este artículo tiene por objetivo analizar algunos aspectos que potenciaron las inundaciones en Colombia en el periodo 2010/2011. La metodología consistió en la revisión y síntesis de información sobre diferentes enfoques para el manejo de inundaciones, en armonía con la visión ecosistémica y de gestión integral de los recursos hídricos. Los resultados indican que las temporadas de lluvias y el fenómeno La Niña de abril de 2010 a diciembre de 2011 dejaron 5,2 millones de personas afectadas y 683 muertos. Dicho evento es el más crítico en la historia, ya que duplica las pérdidas acumuladas por inundaciones en 30 años. Además de lo anterior, se identifica que el desastre se debió a una suma de factores, entre los que destacan: cambios en la cobertura vegetal; las deficiencias en la gestión del suelo y los recursos hídricos; las debilidades en el diseño y gestión de infraestructura, en los patrones de crecimiento demográfico; los problemas institucionales en la prevención y atención de emergencias; la falta de transparencia y la corrupción; una legislación que carece de metodologías para aplicación, de recursos fiscales y de mecanismos para el seguimiento y medición resultados; entre otros. Una de las conclusiones es la necesidad de incluir nuevos conocimientos del clima en la gestión del riesgo y la planificación pero, sobre todo, es recomendable abordar el manejo de las inundaciones con un enfoque diferente, migrando hacia procesos integrales y con mayor capacidad de adaptación de la sociedad colombiana en los próximos años, mediante una gestión del riesgo de inundación más eficiente, acorde a conceptos modernos y en armonía con los procesos de desarrollo sostenible.

\section{PALABRAS CLAVE}

Variabilidad climática, fenómeno ENOS, cambio climático, inundaciones, gestión del riesgo de desastres.

\section{ANALYSIS OF THE ASPECTS WHICH INCREASE THE RISK OF FLOODS IN COLOMBIA}

\section{ABSTRACT}

The main objective of this article is to analyze some aspects that fostered floods in Colombia in the period 2010/2011. The methodology followed was a review and synthesis of information on different approaches to flood management, in harmony with the ecosystemic vision and integrated management of water resources. The results indicate that the rainy season and La Niña phenomenon from April 2010 to December 2011 left 5,2 million people affected and 683 people dead. This has been the most critical event in history, because it doubled the accumulated losses from flooding in 30 years. Besides the above, it has been identified that the disaster was due to a combination of factors, among which are: changes in vegetation cover, 
deficiencies in the management of land and water resources, the weaknesses in the design and management of infrastructure, the population growth patterns, the institutional problems in the prevention and emergency care, lack of transparency and corruption, legislation that lacks enforcement methodologies, fiscal resources and mechanisms for monitoring and measuring the results, among others. One conclusion is the need to include new knowledge on climate, risk management and planning, but above all, it is recommended to address the management of floods with a different approach, migrating to more comprehensive processes and with a higer adaptation capacity from the Colombian society in the coming years through a more efficient flood risk management, in accordance with the modern concepts and in harmony with sustainable development processes.

\section{KEY WORDS}

Climate variability, ENSO phenomenon, climate change, floods, disaster risk management.

\section{ANÁLISIS DE ASPECTOS QUE INCREMENTAN EL RIESGO DE INUNDACIONES EN COLOMBIA}

Las inundaciones y el desarrollo son temas relacionados; antiguas civilizaciones como la India, China y Egipcia, aprovecharon las crecidas para generar abundancia y fertilidad. En América, las sociedades también se adaptaron y manejaron el territorio en condiciones ambientales adversas; Aztecas, Chibchas y Zenúes, habitaron zonas inundables y mediante técnicas de manejo del agua y la pesca, sostuvieron la población indígena en lo que actualmente es México y Colombia; modificando levemente el paisaje gracias a su comprensión sobre la naturaleza de las inundaciones y de los beneficios que ofrecían. Recientemente, es normal atribuir las catástrofes por inundaciones a la Variabilidad (VC) y al Cambio Climático (CC), porque se vienen presentando frecuentes anomalías de precipitación (Vincent, 2007; Brown \& Funk, 2008) que impactan ecosistemas y sectores socioeconómicos, con pérdidas humanas y económicas (Carvajal-Escobar, 2010, 2011a, 2011b). Establecer la frontera entre VC y CC es difícil en regiones de marcada influencia de la primera, en las condiciones normales de clima, como es el caso de Colombia donde la hidroclimatología está fuertemente influenciada por el fenómeno El Niño Oscilación del Sur (ENOS) (OMM, 2009). Sin embargo, los cambios del clima no son la única causa y se requieren explicaciones adicionales.

Mediante un análisis, revisión y síntesis de información relevante sobre el tema, se presenta una reflexión sobre factores antrópicos que contribuyeron a las inundaciones, bajo la hipótesis de que aunque el fenómeno La Niña fue intenso, no fue la única causa de la catástrofe en Colombia, porque aspectos tales como: la prevención del riesgo en la planificación de los proyectos de reconstrucción, la construcción social del riesgo, y una mayor preocupación por las comunidades más afectadas, son aún invisibles en muchos planes de gobierno local y regional. El reconocimiento de la vulnerabilidad como causa principal de las pérdidas, permitiría identificar acciones para reducirla en un contexto de desarrollo sostenible, como lo plantean los Objetivos de Desarrollo del Milenio. El crecimiento de sociedades que no aseguran la preservación ambiental y la equidad social, potencian la ocurrencia de desastres y dejan amplios sectores con baja 
capacidad de respuesta ante eventos catastróficos, situación que es más dramática en Colombia ya que alcanza niveles del 34\% en pobreza (DANE, 2011). La idea tradicional de gestionar riesgo y adaptación centrándose en la infraestructura y no en las personas, es un planteamiento persistente a revisar en muchos planes de reconstrucción.

Las temporadas de lluvias 2010-2011 y el fenómeno La Niña, dejaron 5,2 millones de personas afectadas (11,3\% de la población del país) en 1.027 municipios (87\%), incluyendo el Distrito Capital. Las áreas de cultivo anegadas superaron el millón de hectáreas y las pérdidas económicas sobrepasaron los US \$4.870 millones (Gómez, 2011 ; Swiss Re, 2011; UNGRD, 2011).

\section{METODOLOGÍA}

\section{Descripción de la zona de estudio}

Colombia está localizada en la zona ecuatorial (Figura 1); por su cercanía a los océanos y formas de relieve, entre otros aspectos, presenta gran variedad de climas, suelos y disponibilidad hídrica, a lo cual se suma una amplia diversidad biológica, étnica y cultural (IDEAM, 2001).

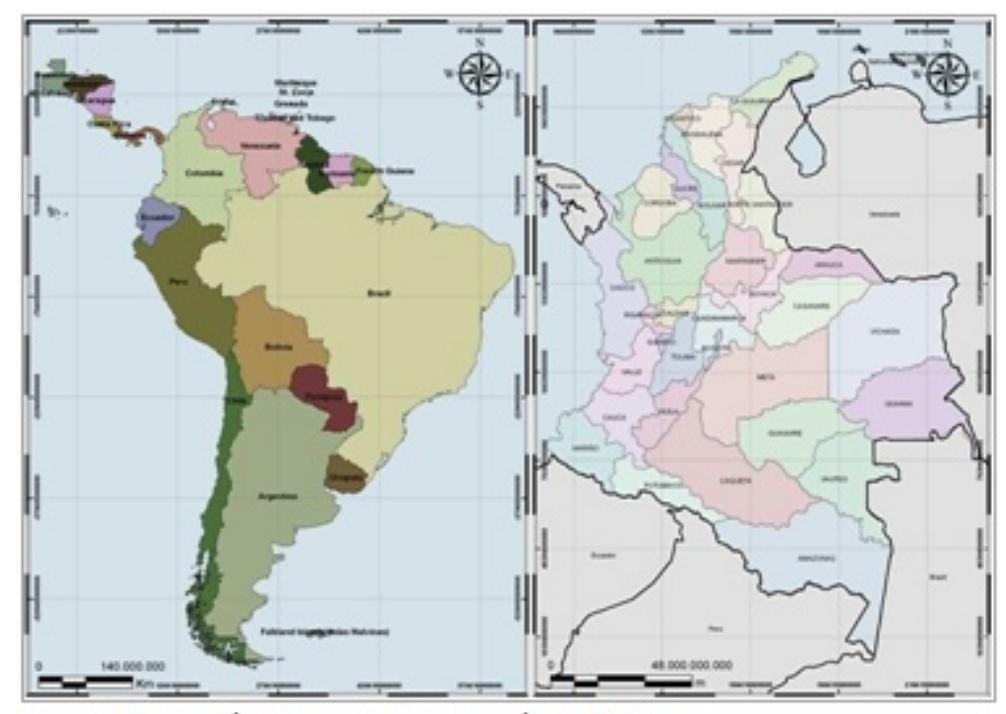

Fuente: Elaboración Grupo de Investigación IREHISA.

Figura 1. Localización de la zona de estudio.

Alrededor del $70 \%$ del territorio presenta lluvias anuales promedio superiores a $2.000 \mathrm{~mm}$; en la costa Pacífica estas superan los 12.000 mm/año, mientras en el norte, el clima es desértico. La precipitación intraanual presenta dos regímenes característicos: bimodal en la zona Andina, cuyos trimestres lluviosos son marzo-abril-mayo (MAM) y septiembreoctubre-noviembre (SON); y regímenes mono-modales en la parte oriental de Colombia (Puertas \& Carvajal-Escobar, 2008). 


\section{Revisión bibliográfica y análisis de información}

La búsqueda sistematizada de información en bases de datos como Web of Science, Redalyc y Dialnet, ofrece documentación pertinente y relevante sobre el tema. Autores como Tompkins \& Adger (2005) y Janssen et al. (2006) han utilizado la búsqueda sistematizada para mejorar la probabilidad de ubicar documentos relevantes sobre la adaptación al CC, apoyándose en la preselección de criterios de búsqueda como son las fuentes de información y las palabras clave. En este caso, los criterios de búsqueda incluyen artículos científicos, informes periodísticos, documentos institucionales y libros publicados. La mayoría de publicaciones tenidas en cuenta se desarrollaron en los últimos 20 años. Se seleccionó un listado de conceptos clave para acotar la búsqueda, el resultado obtenido es información general asociada a los objetivos como: considerar múltiples enfoques para la gestión de inundaciones, la reducción del riesgo de desastres, abordar la visión ecosistémica en la gestión integral de los recursos hídricos y analizar algunos factores que contribuyen a las inundaciones en Colombia.

Otro tipo de base de datos consultadas como Em-Dat, SNPAD y DesInventar, permiten caracterizar las inundaciones dentro de las estadísticas globales y nacionales. Este tipo de información, puede utilizarse para apoyar la toma de decisiones, comparar, resumir lecciones aprendidas y proveer información de fácil comprensión a distintos usuarios. Szlafsztein et al. (2007), Aldunce et al. (2008) y Debels et al. (2009) definen varios tipos de sistematización y caracterización para levantar y difundir información.

\section{EVALUACIÓN DE RESULTADOS}

Colombia está caracterizada por sus tres cordilleras y áreas de planicie inundable. Tiene un ciclo hidrológico dinámico al estar ubicada en el trópico ya que el predominio de la Zona de Confluencia Intertropical y los procesos que ocurren en los océanos Pacífico, Atlántico y mar Caribe, ocasionan variaciones en la distribución espacio-temporal de la precipitación, de la temperatura y de otras variables climatológicas.

El volumen de agua disponible anualmente en Colombia es de $2.100 \mathrm{~km}^{3}$ y representa el 4,5\% del agua mundial, situando al país entre los primeros 10 productores del líquido (IDEAM, 2004). Teniendo en cuenta que la superficie periódicamente inundable es de 11 millones de ha (es decir el $9,2 \%$ del territorio), es de prever que los desastres más frecuentes y de mayor impacto económico sean las inundaciones. Además de lo anterior, la VC juega un papel clave en el comportamiento de los ríos porque el fenómeno La Niña puede generar aumentos en los caudales mensuales de los ríos de hasta un $60 \%$ en los trimestres más lluviosos (IDEAM, 2010). Cabe destacar que por efecto del CC se incrementará la frecuencia de ocurrencia de eventos extremos del fenómeno ENOS, que afectarían considerablemente la región (Collins et al., 2005; Magrin et al., 2007).

El impacto generado por las temporadas de lluvias y el fenómeno La Niña desde abril de 2010 hasta diciembre de 2011 fue: 5'208.504 personas afectadas y 683 muertos; daños en 1.027 municipios y en 28 de 32 departamentos, incluido el Distrito Capital (Semana.com, 2011). Se 
inundaron 1'080.000 ha de cultivos, pastos y centros urbanos. En general, el 11,3\% de los colombianos padecieron los daños (Colombiassh.org, 2011; SNPAD, 2011 ; UNGRD, 2011). EI BID y la CEPAL (2010) catalogaron las inundaciones dentro de las catástrofes con mayor número de víctimas mortales con un costo por daños totales que ascendió a más de US $\$ 4.870$ millones (Gómez, 2011a).

Los desastres por inundaciones desde 1970 hasta el año 2000 han tenido un costo para Colombia del 2,2\% del PIB (US $\$ 2.000$ millones del año 2000) (ONU, BID y CEPAL, 2007 ). Este último evento ha sido el más crítico en la historia por los daños, ya que casi duplica las pérdidas acumuladas por inundaciones en 30 años. El desastre se debe a una suma de factores, en su mayoría de carácter antrópico.

La Figura 2, muestra el área inundada en proporción al tamaño del departamento y los tipos de bienes afectados. Los mayores daños sobre cultivos se presentaron en la región Andina, y la costa Atlántica, donde se encuentran las llanuras de inundación de los ríos Magdalena y Cauca (Bajo Magdalena y Depresión Momposina, respectivamente), también muestra que debido a las características de urbanismo en Colombia, hubo un gran impacto sobre las viviendas, con efectos intensos en la región Pacífica y la costa Atlántica.

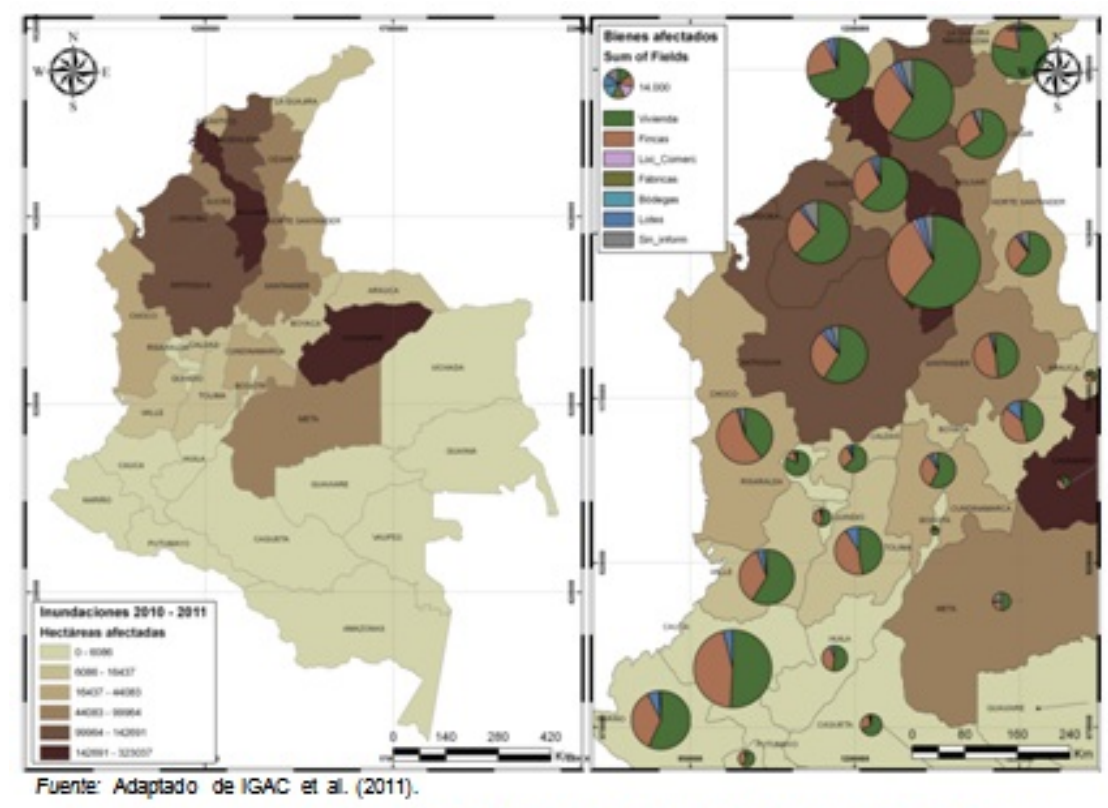

Figura 2. Inundaciones en Colombia y bienes afectados en el periodo 2010-2011.

\section{¿A QUÉ SE DEBE QUE LAS INUNDACIONES DEL 2010-2011 SE HALLAN CONVERTIDO EN UNA CATÁSTROFE?}

Dentro de las explicaciones a la gravedad de las inundaciones están la VC, ya que las oscilaciones climáticas durante el año 2010 resultaron ser severas sobre las zonas del país con mayor influencia del fenómeno ENOS, lo que aumentó los niveles de caudal de los ríos, hasta los más altos 
registrados históricamente (IGAC et al., 2011). El evento La Niña 20102011, se clasifica como el segundo más crítico en 51 años, a partir del Índice Multivariado ENOS (MEI) (Wolter \& Timlin, 1993, 1998, 2011).

De acuerdo a los valores del MEI (Figura 3), los eventos más intensos se han registrado en los años 1954, 1964, 1970, 1973, 1988, y 2010-2011, este último alcanzó valores extremos en los trimestres sep.-oct.-nov./2010 y ene.-feb.-mar./2011.

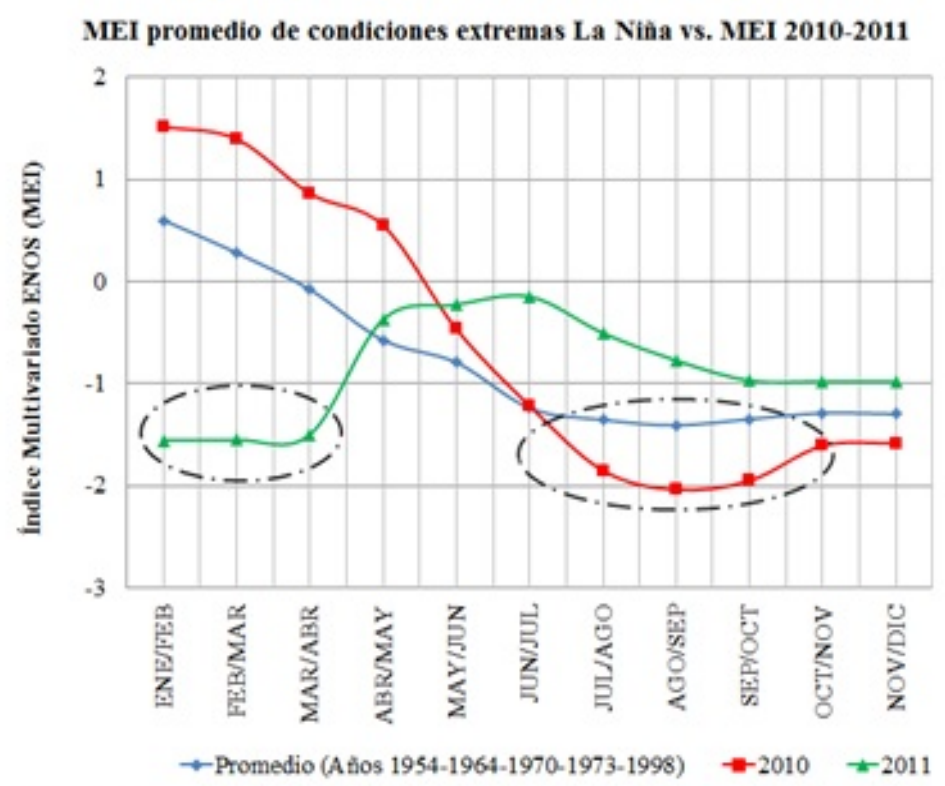

Fuente: Adaptado de Wolter \& Timlin (2011).

Figura 3. Promedio del MEl, para los últimos 5 eventos más fuertes de La Niña vs. condiciones del MEI 2010-2011.

Teniendo en cuenta que los mayores efectos de La Niña sobre las precipitaciones en zona Andina colombiana se presentan un mes después de su inicio, y entre dos y tres meses después en los caudales medios de los grandes ríos (Carvajal-Escobar, 2004), es posible relacionar el desastre presentado con el fenómeno La Niña, al observar los picos bimestrales de población afectada que ocurrieron en los trimestres típicamente lluviosos de 2010-2011 y que en el año 2010 se extendieron a JAS (trimestre normalmente seco) en la región Andina (Figura 4). 


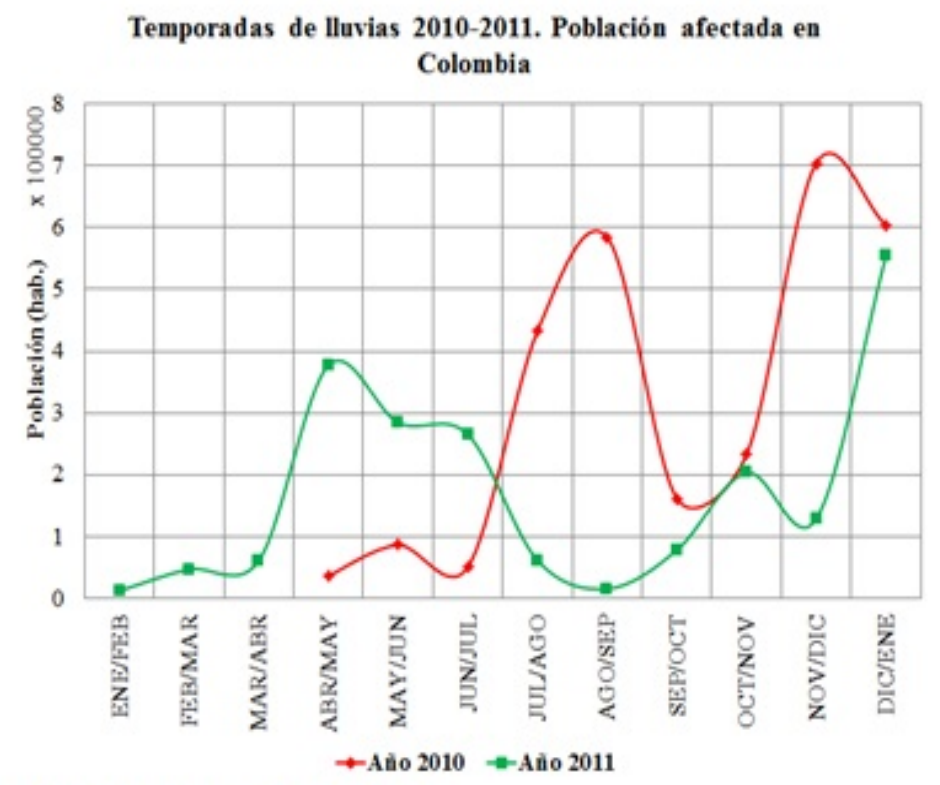

Fuente: Adaptado de SNPAD (2011).

Figura 4. Personas afectadas por la temporada de lluvias intensas, abril 2010-diciembre 2011.

La Niña, ha ocurrido 13 veces en los últimos 60 años, con una frecuencia media de siete años (Carvajal-Escobar, 2011a). Sin embargo, a partir del análisis de información sobre Colombia en bases de datos sobre desastres (DesInventar.org, 2011), las emergencias por inundaciones con más de 500 mil personas afectadas ocurren en promedio cada dos años en los últimos 20 años. Esto muestra dos aspectos: primero, que nuestras comunidades deberían estar mejor preparadas para las intensas lluvias y los desbordamientos de los ríos; y segundo, que no se necesita un evento La Niña para generar calamidades. De esta forma, se puede inferir que además de la VC hay otros factores asociados al desastre 2010-2011 como: la reducida gestión del riesgo de sociedades ubicadas en zonas inundables o de fuerte pendiente, donde no se da la importancia necesaria a los procesos de preparación y prevención, o las instituciones involucradas no dan continuidad a las políticas sobre el tema.

Otra razón que explica la magnitud de las inundaciones, es el deterioro de la cobertura vegetal en las zonas altas y medias de las cuencas, ya que las propiedades del suelo y las condiciones de saturación previas a una precipitación son factores determinantes en la producción de la escorrentía posterior a una lluvia. Por lo tanto, la deforestación, la impermeabilización, la desecación de humedales y la expansión de la agricultura son factores que aumentan el riesgo de inundaciones, porque contribuyen al aumento de la escorrentía en los ríos, al depósito de sedimentos en los cauces y a la desestabilización de suelos saturados, que pueden generar represamientos $\mathrm{y}$ posteriores avenidas torrenciales que afectan poblaciones $\mathrm{e}$ infraestructura vial. A escala nacional, la tasa de deforestación anual estimada en el periodo 2000-2007 fue de 336.000 ha, ubicando a Colombia en el octavo lugar entre los países con mayor deforestación en el mundo (Duque, 2011).

Las deficiencias en la gestión del suelo, de los recursos hídricos y el inadecuado ordenamiento territorial, también hacen que las inundaciones 
se transformen en catastróficas. La urbanización sobre el lecho mayor de los ríos, la modificación de la forma de los cauces y la alteración de los ciclos vitales de los ecosistemas, derivan en inundaciones más frecuentes y de mayor magnitud. Estas modificaciones aparecen debido a la expansión de la minería, la generación de energía y la urbanización. Hasta el momento en Colombia, la gestión del suelo y de los recursos hídricos se maneja de forma fragmentada, sin considerar el papel de las inundaciones en el ecosistema. Adicionalmente, la cuenca aún no se interpreta considerando la interacción suelo-agua-clima-sociedad. Esto se debe en parte a la gestión de los recursos naturales dentro de límites administrativos y de gobierno que no coinciden con los límites naturales de las cuencas, resultando en un traslape de responsabilidades a diferentes escalas de gobernabilidad y una falta de coordinación entre instituciones en las zonas de frontera.

Las debilidades en la gestión y administración de las obras de infraestructura, encauzamiento, protección y regulación de los ríos, son otras causas de la gravedad de las inundaciones. En el país, como en otros países en desarrollo, las labores de operación y mantenimiento de la infraestructura son limitadas o inexistentes porque dependen de un engranaje interinstitucional debilitado por la política, la corrupción y la burocracia. Aunque existen estudios para actualizar y mejorar la infraestructura, el acceso a los recursos para ejecutarlos es lento y complejo. La situación de orden público en algunas regiones, la privatización de entidades y la tercerización de labores de operación y mantenimiento, dificultan la adecuada gestión de obras. En resumen, no existe un plan de manejo continuo y completo de la infraestructura.

Otro factor adicional, está asociado al desarrollo de obras civiles, y la relativa garantía de protección frente al riesgo de inundaciones de las estructuras, amparadas en métodos de diseño de hace más de 50 años, cuyos resguardos, factores de seguridad y métodos de estimación de la probabilidad de ocurrencia de un evento extremo, no son suficientes para proteger a las sociedades de los riesgos asociados a la VC y al CC.

Muchas de las grandes obras hidráulicas del país fueron construidas hace varias décadas y sus diseños no consideraron los cambios recientes y los problemas ambientales emergentes, tales como: aumento de deforestación, desecación de humedales o zonas naturales de amortiguamiento, cambios en el uso del suelo y en el clima, etc. En la actualidad, la gestión del agua, del riesgo, y la forma de abordar problemáticas ambientales han cambiado rápidamente, exigiendo la revisión del enfoque tradicional de diseño (que analiza eventos independientes y aislados), a otros más modernos centrados en: el análisis de eventos recurrentes que consideren la combinación de amenazas, las condiciones antecedentes y el estudio de sistemas hídricos completos en condiciones críticas, entre otros.

Los eventos extremos asociados a ENOS son cada vez más frecuentes y vienen acompañados de mayor incertidumbre, lo cual invita a hacer una reflexión sobre el daño que puede ocasionar una obra cuando se presentan fallos. Es pertinente avanzar aún más en: normas de diseño, en ajustes metodológicos, y en el análisis de la incertidumbre asociada a la reducción de inundaciones ordinarias que puede aumentar el riesgo de desastres ante desbordamientos extremos, al generar una falsa sensación de seguridad por el poco uso de las estructuras de protección; lo cual incentiva una mayor ocupación de zonas de riesgo, deteriora la conciencia social, y 
puede llevar a la reducción u omisión de presupuesto para mantenimiento adecuado, o para el financiamiento de acciones complementarias en la Gestión del Riesgo (Carvajal-Escobar, 2011a).

La ingeniería debe ir más allá de trasladar la inundación de un lugar a otro en la cuenca, de retener los volúmenes de escorrentía pluvial, de movilizar rápidamente los flujos de agua o aumentar la capacidad de transporte de los cauces, sobre todo porque las proyecciones frente al CC muestran una tendencia hacia la desertificación en las áreas donde las lluvias extremas están produciendo inundaciones catastróficas. Los enfoques modernos buscan desarrollos técnicos que se adapten mejor al ambiente o imiten el comportamiento natural de los ríos, aprovechando así los bienes y servicios que prestan los ecosistemas, mediante la recuperación de áreas de laminación natural (llanuras de inundación, humedales, áreas de recarga de acuíferos, etc.), disminución de las demandas de agua, alargando al máximo posible su ciclo dentro de las comunidades, o mediante la conservación de las condiciones naturales de las cuencas. Todo lo anterior, debe ir acompañado de medidas no estructurales que promuevan la sostenibilidad ambiental y el beneficio económico, que incluyan procesos de fortalecimiento de capacidades a todo nivel, incluyendo a las sociedades que habitan zonas de riesgo, para adaptarse y sostener en el tiempo actitudes de prevención y mejor respuesta ante eventos de inundación, que rompan el ciclo de desastre, reparación, olvido y nuevo desastre (SedanoCruz, 2012).

Actualmente, las periferias marginales ocupan un porcentaje importante de las grandes ciudades de Suramérica, como Río de Janeiro, Buenos Aires, Bogotá, etc., además las tendencias globales dicen que al 2030 el 60\% de la población mundial vivirá en áreas urbanas (UN/DESA, 2005 ). El desplazamiento desde la zona rural, ya sea por búsqueda de oportunidades o situaciones de conflicto armado, está aumentando la construcción social del riesgo en Colombia. La población se ha cuadruplicado en los últimos 50 años. Sin embargo, la cobertura de servicios e infraestructura en las ciudades no aumenta al mismo ritmo. Los municipios, aunque deben definir áreas aptas para el urbanismo, no tienen cómo garantizar el acceso a vivienda a la población de baja renta que se encuentra en acelerado crecimiento. Por lo tanto, las comunidades se expanden sobrepasando las fronteras del territorio "seguro" -ocupando áreas de riesgo de inundación o deslizamiento- sin planificación, apoyo institucional o lineamientos técnicos. Todo lo anterior, incrementa la vulnerabilidad con lo que es posible que se amplifique la magnitud de los desastres, sin necesidad de que se manifiesten amenazas hidro-meteorológicas extremas como ENOS.

La debilidad de las instituciones en la prevención y atención de desastres, es quizás uno de los principales aspectos que influyó en la gravedad de la emergencia a nivel nacional. Desde lo técnico se detectan deficiencias en los Sistemas de Alerta Temprana, y la cobertura de estaciones de monitoreo hidro-meteorológico no es suficiente para modelar o pronosticar a escalas regional y local. A pesar de contar con un Sistema Nacional para la Prevención y Atención de Desastres constituido desde el año 1989, 808 de 1041 municipios (78\%) carecen de dependencias para el manejo de emergencias. Los Planes de Ordenamiento Territorial (POT) siguen siendo modificados en sus límites de expansión urbana, permitiendo usos del suelo inadecuados. La compleja red de instituciones que manejan el ambiente, los recursos naturales y el desarrollo económico siguen considerando la cuenca hidrográfica de forma fragmentada con visiones distintas que dificultan las soluciones integrales. 
La falta de transparencia y la corrupción son un aspecto más del problema. La emergencia mostró que muchas de las obras estaban mal construidas, había contratos mal ejecutados, desviación de dinero, etc., producto de la política electoral y de la deficiente gestión de alcaldes, gobernadores y directivos de las Corporaciones Autónomas Regionales. Posterior a la emergencia y en época de campañas electorales, la Procuraduría y la Contraloría (entes autónomos de control y vigilancia de las funciones de empleados del Estado y del manejo de los recursos públicos), empezaron a investigar casos de fraude y a alcaldes que no formularon proyectos pertinentes a la recuperación posterior a los desastres.

Aunque existe legislación para proteger los usos del suelo, los recursos hídricos y el ordenamiento territorial, muchas instituciones no la hacen cumplir debido a: falta de metodologías para su adecuada aplicación, carencia de garantías y de recursos fiscales para ejecución, y debilidades para el seguimiento y medición de sus efectos en la calidad de vida de la sociedad. Un ejemplo de lo anterior se presentó durante el desastre 20102011, donde ante las grandes dificultades de las instituciones y las misiones interagénciales para determinar el número de damnificados, cuantificar los daños y establecer las necesidades inmediatas, la entidad que encabezó la gestión de la emergencia (Colombia Humanitaria) se limitó a dar reportes financieros, desaprovechando la oportunidad para hacer una gestión eficaz (Oxfam, 2011 ).

Un aspecto que agravó la emergencia fueron los problemas con las ayudas entregadas que resultaban insuficientes, y en algunos casos no respondían a las características culturales y/o necesidades de los diferentes grupos que componen la población. Un ejemplo de lo anterior es el papel de la mujer como población vulnerable. En muchos lugares las mujeres se hacen responsables de las condiciones de salud, alimentación, cuidado de niños y ancianos, por tanto para ellas resulta urgente resolver este tipo de problemas. Además, los hombres sienten como primera necesidad la recuperación de sus viviendas, por lo que herramientas y conocimientos para reconstruir sus hogares, en muchos casos, son lo más importante. Los ejemplos anteriores muestran la necesidad de incorporar el enfoque de género en la gestión integrada del recurso hídrico, sobre todo porque múltiples autores reportan que: las mujeres han sido capaces de movilizar a la comunidad en las diferentes fases del ciclo de gestión del riesgo (GuhaSapir, 1997; Shrader \& Delaney, 2000; GWA \& WSP, 2005; Yonder et al., 2005); la sociedad tiene conocimientos importantes que aportar; y su empoderamiento involucra mejores mecanismos de revisión, transparencia, e inclusión (Carvajal-Escobar \& Quintero, 2007).

\section{¿QUÉ REFLEXIONES QUEDAN?}

Las inundaciones hacen parte del ciclo hidrológico, siempre han existido y la humanidad ha aprovechado sus beneficios para la fertilidad de suelos y la biodiversidad de los ecosistemas conexos como: humedales, lagunas, etc. No obstante, con el paso del tiempo, el desarrollo y la ocupación del territorio hacen que las inundaciones se asocien más a catástrofes, a la VC y al CC; y aunque es cierto que la fluctuación natural y/o antrópica del clima juega un papel muy importante, también lo juegan los procesos en los cuales se busca dominar la naturaleza en lugar de convivir con ella (Sedano et al., 2011). Es necesario replantear, en la medida de lo posible, 
soluciones de ingeniería que respeten el espacio de los cursos de agua, y restaurar a condiciones más parecidas a las naturales permitiendo que se den mecanismos de autorregulación de picos de caudal.

Las inundaciones son un problema para los países en desarrollo. El acelerado crecimiento de la población en los últimos 50 años, ha estado acompañado por problemas de gobernanza y de planificación territorial, se ha permitido la ocupación de áreas con alto riesgo a desastres socionaturales; en el caso colombiano se acompaña además de otros problemas como el poco control de la degradación ambiental, la inadecuada ejecución de obras civiles, y las dificultades de orden público que hace más difícil la atención de emergencias.

Es necesario replantear la visión de los gobernantes sobre la prevención del riesgo de inundaciones, las partidas presupuestales y la importancia de los programas emprendidos para fortalecer las capacidades de adaptación ante desastres; especialmente en un país con múltiples amenazas como Colombia, donde es necesario fortalecer la gestión integrada de riesgos en el manejo del territorio y de los recursos hídricos.

Cada que ocurre una inundación se produce un shock sobre las proyecciones de crecimiento económico. De acuerdo a la renta de un país, existen diferencias en las tendencias sobre los impactos en mortalidad y pérdidas económicas en términos absolutos; ya que por cada dólar que se pierde a causa de una catástrofe en un país de baja renta, se pierden alrededor de 16 dólares por la misma situación en los países con mayor poder adquisitivo. Lo opuesto sucede en las estadísticas sobre mortalidad en esta clasificación de países. Sin embargo, cuando las pérdidas económicas son estandarizadas en términos de desarrollo económico o PIB, ambos -la mortalidad y el impacto económico- se incrementan en la medida en que las economías son más pobres (Hoyois \& Guha Sapir, 2012).

La Figura 5, refleja de forma conceptual lo que sucede en el país cuando se presenta una inundación. En primer lugar, se genera una caída en la curva de crecimiento pero, a raíz de las inversiones nacionales y extranjeras (para atención de emergencias y recuperación), se presenta un crecimiento acelerado. Sin embargo, si la sociedad no tiene las condiciones de resiliencia y adaptación suficientes, puede ocurrir que no se logren alcanzar los niveles de desarrollo proyectados previamente a la manifestación del desastre. 


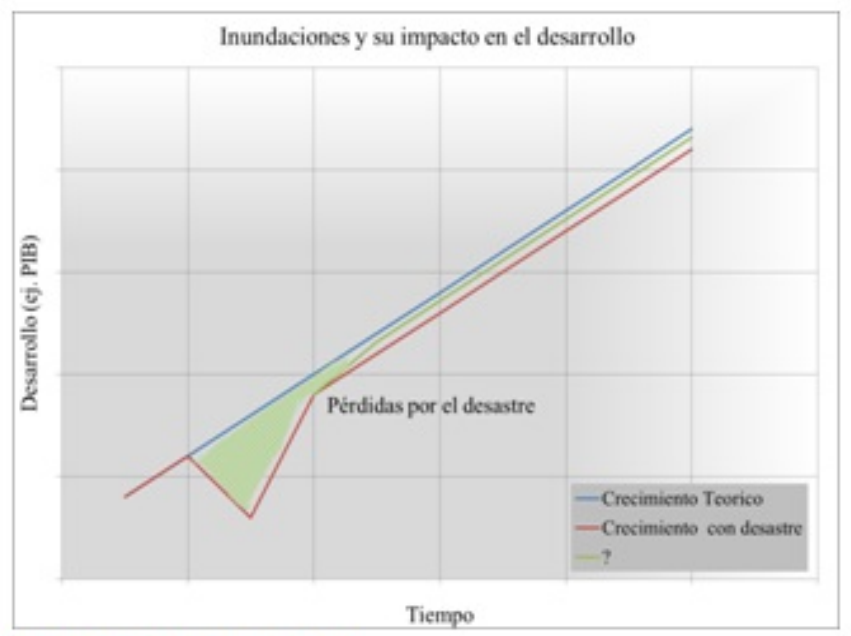

Fuente: Brandimarte (2012).

Figura 5. Representación conceptual del posible impacto de las inundaciones en el desarrollo económico de una sociedad hipotética.

Asociar directamente el crecimiento económico y el impacto de las inundaciones en Colombia, no es posible y no hace parte del alcance de este trabajo. Otros factores como el conflicto interno, el comportamiento de los sectores productivos, las políticas de gobierno, otros desastres socionaturales, etc., tienen efectos fuertes en el comportamiento del PIB. No obstante y a manera de ejemplo, se puede hacer cruzar la información de crecimiento económico y las inundaciones presentadas en los últimos 10 años en Colombia (Figura 6). Si se descarta del análisis el periodo 20082009 que corresponde a la crisis financiera global, y se adopta como fuerte impacto por inundaciones aquellos años donde hay más de un millón de personas afectadas; se observa: en 2004-2005 un decrecimiento en la economía del país, con una posterior recuperación en el periodo 2005-2006 (semejante al comportamiento teórico de la Figura 5), y un cambio de pendiente en el periodo 2010-2011 -donde el ritmo de crecimiento disminuyó, influenciado por muchos aspectos, entre los cuales se manifiesta la gravedad de las inundaciones que tienen un costo proyectado de $0,5 \%$ del PIB en 2011 y comprometen recursos del Gobierno Nacional Central hasta el año 2014- (DNP, 2011; MinHacienda, 2011). Sin embargo, el crecimiento económico sigue con proyección positiva ya que la formulación de nuevos impuestos sobre el patrimonio, la minería, la producción energética y el comercio, afortunadamente han evitado pérdidas más visibles en la curva (BanRepublica.gov.co, 2011). 


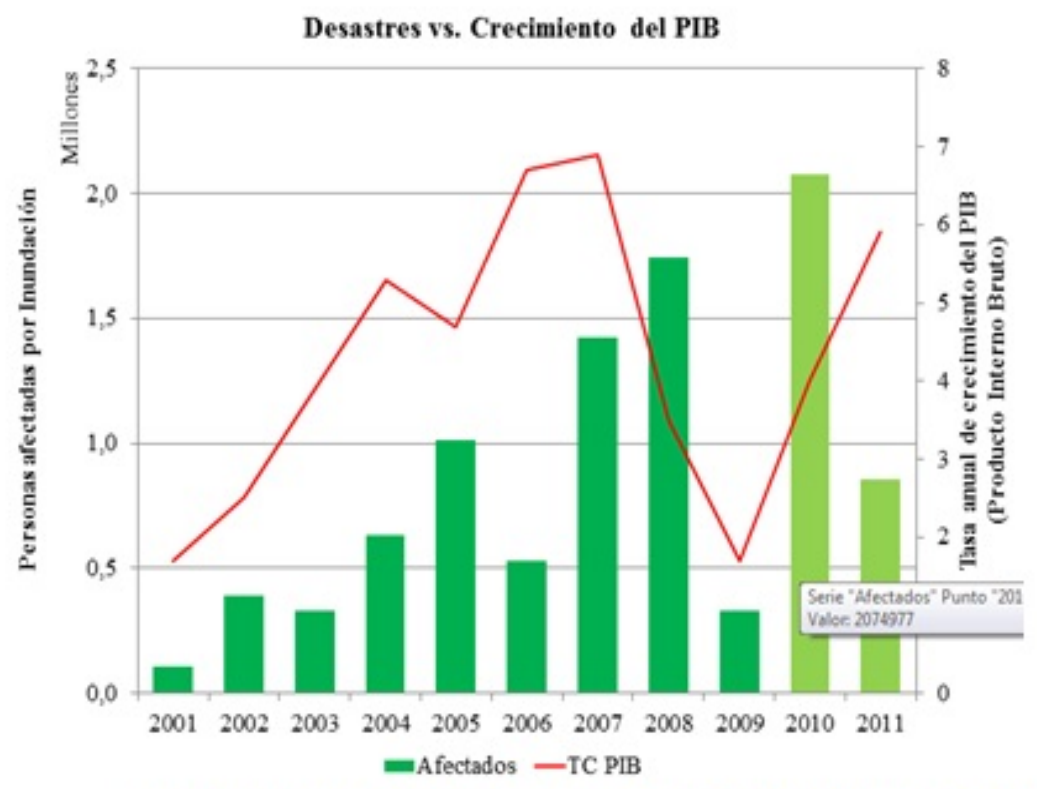

Fuente: Adaptado de Desinventar.org (2011) y BanRepublica.gov.co (2012).

Figura 6. Variación del crecimiento en Colombia y las tendencias sobre afectados por inundaciones.

Los problemas asociados a inundaciones y al deterioro de los recursos hídricos, van más allá de falta de coordinación entre instituciones; están más asociados a fronteras institucionales, a burocracia y a traslape de responsabilidades. La falta de planeación deja vacíos que no son atendidos por el Estado. Por lo tanto, las nuevas propuestas de planeación deben contemplar bases conceptuales que integren el sistema suelo-agua-climasociedad. Incluyendo siempre la gestión del riesgo de desastres socionaturales y múltiples riesgos (amenazas naturales y conflicto armado, por ejemplo).

\section{CONCLUSIONES Y RECOMENDACIONES}

Un aporte de la investigación, es la caracterización de algunos factores que agudizan los desastres por inundaciones en Colombia; hasta el momento la información disponible sobre el tema se encuentra dispersa, es incompleta, es inadecuada y/o el acceso a la misma es limitado. Por lo tanto, este trabajo contribuye a visibilizar un problema que no se ha incluido en los Planes Nacionales de Desarrollo, pero que tiene un fuerte impacto sobre la calidad de vida de las personas y del progreso de las regiones. Hasta el año 2010, las inundaciones se habían considerado un desastre menor porque se comparaban con la capacidad de daño que producen otros fenómenos como la sismicidad y el vulcanismo. No obstante, este trabajo muestra que es el desastre socio-natural, con mayor número de personas afectadas a lo largo de la historia.

La variabilidad climática y el cambio climático plantean problemas significativos, ya que las condiciones hidro-meteorológicas están cambiando, incrementando la incertidumbre en la gestión de los recursos hídricos. Esto implica modificar el enfoque tradicional de la gestión de 
inundaciones y pasar de "estar absolutamente a salvo" a una idea más flexible y con mayor capacidad de adaptación para "vivir con el riesgo de inundaciones", además de otros riesgos que pueden intensificar los desastres socio-naturales, puesto que los cambios en el clima generan impactos en la producción agrícola y en las planicies de inundación urbanizadas; y porque el futuro crecimiento de la población ejercerá todavía más presión sobre los recursos naturales.

Resulta contradictorio que las regiones hoy amenazadas por eventos extremos de inundación, en el futuro sean amenazadas por las sequías previstas según los modelos de CC. Esto significa que la gestión integrada de las inundaciones no puede enfocarse solo en grandes obras estructurales, sino en la adaptación y gestión del riesgo. Un aspecto clave sobre las obras de protección, es la validez de la información técnica necesaria para desarrollar obras robustas y duraderas. Aunque el diseño tradicional funcionó antes para disminuir el riesgo de inundaciones, en la actualidad se vienen presentado fallos debidos al impacto ambiental, a su corta sostenibilidad y a los costos de su manejo. Las nuevas tendencias deben procurar desarrollos técnicos que se adapten mejor al ambiente.

La política colombiana debe procurar articular la legislación vigente hacia la visión del modelo de desarrollo sostenible, manejando una visión holística de la cuenca, de los recursos hídricos y el papel de la sociedad dentro del ciclo hidrológico; la necesidad de desarrollo económico parece estar siempre en oposición a la protección ambiental de los recursos ambientales, pero se necesita determinar hasta dónde llevar la productividad del país, a costo de la disminución de la capacidad de resiliencia de la sociedad, la naturaleza y el incremento de la vulnerabilidad de las personas frente a los desastres.

El análisis del comportamiento hidrológico de la cuenca debe realizarse con metodologías que incluyan el uso de variables macroclimáticas [ej. Multivariate Enso Index (MEI), Southern Oscillation Index (SOI) y Oceanic Niño Index (ONI)] porque permiten incluir el impacto de ENOS (Puertas \& Carvajal-Escobar, 2008; Bedoya et al., 2010). Por ejemplo, en cuencas torrenciales andinas del departamento del Valle del Cauca, la aplicación de estas variables permitió establecer que el fenómeno ENOS tiene mayor correlación con el comportamiento de caudales que con la precipitación (Ávila, 2012; Gutiérrez, 2013). A partir de este tipo de estudios, es posible establecer medidas de gestión de los recursos hídricos para reducir las pérdidas que actualmente ocasiona este tipo de eventos extremos, y conllevan a una mejor adaptación frente a la VC y al CC -en casos específicos de inundaciones y sequías- en el entorno hídrico de consumo humano y de generación de energía, que son los dos principales usos que se le dan al agua en esta zona del país.

Aunque en Colombia los desastres por inundaciones ocurren con mucha frecuencia, la falta de información científica, técnica y social representa en sí misma un riesgo. Se evidencian avances en la vigilancia y análisis de las amenazas a escala nacional, pero no se dispone de información suficiente y detallada que permita efectuar análisis adecuado a escalas más pequeñas y locales. 


\section{AGRADECIMIENTOS}

Al grupo de Investigación en Ingeniería de Recursos Hídricos y Suelos IREHISA ${ }^{4}$ - de la escuela EIDENAR, Universidad del Valle. A Colciencias, Programa de Becas Francisco José de Caldas. A la Universidad Politécnica de Valencia. Y al proyecto "Cuencas claves en Colombia para la implementación de la nueva política de la gestión integrada del recurso hídrico", COLCUENCAS, Ministerio de Ambiente y Desarrollo, Universidad del Valle, IDEAM y UNESCO-IHE, que apoyaron con los recursos necesarios para la realización de este documento.

\section{BIBLIOGRAFÍA}

- Aldunce, P.; Carvajal-Escobar, Y.; León, A.; Neri, C.; Quintero, M. y Soza, S. (2008). Sistematización de las políticas y estrategias de adaptación nacional e internacional al cambio climático del sector silvoagropecuario y de los recursos hídricos y edáficos. Informe Final. p. 132. Obtenido de: http://www.odepa.gob.cl/odepaweb/ servicios-

informacion/publica/Estudio_adaptacion_cambio_climatico.pdf

- Ávila, A. (2012). Simulación hidrológica Utilizando HEC-HMS como herramienta para estimar caudales máximos en la cuenca hidrográfica del Río Cali. Tesis de pregrado, Universidad del Valle, Cali, Colombia. No publicada.

- BanRepublica.gov.co. (2011). Informe a la junta directiva del Congreso de la República de Colombia - Marzo 2011. Obtenido de: http://www.banrep.gov.co/documentos/publicaciones/ informe_congreso/2011/marzo.pdf

- _ (2012). Boletin de indicadores económicos. Colombia. Obtenido de: http://www.banrep.gov.co/documentos/seriesestadisticas/ informes/bie.pdf

- Bedoya, M.; Contreras, C. y Ruiz, F. (2010). Alteraciones del régimen hidrológico y de la oferta hídrica por variabilidad y cambio climático. En: Estudio Nacional del agua 2010 (pp. 282-320). Colombia: Instituto de Hidrología, Meteorología y Estudios Ambientales IDEAM-. Obtenido de: https://documentacion.ideam.gov.col openbiblio/Bvirtual/021888/ENA2010Cap7.pdf

- Brandimarte, L. (2012). Inundaciones urbanas, nuevas oportunidades, incertidumbres y gestión. [Diapositivas de ppt.] En: Curso Internacional Gestión Integrada del Riesgo por Inundaciones: Conceptos, Medidas y Desafíos. Unesco-HIDROEX. Octubre-2012, Cali, Colombia.

- Brown, M \& Funk, C. (2008). Climate - Food security under climate change. Science, 319(5863), 580-581.

- ONU.; BID y CEPAL. (2007). Información para la gestión del riesgo de desastres. Estudios de caso de cinco paises. Caso de Estudio Colombia. Obtenido de: http://www.cepal.org/colombia/noticias/documentosdetrabajo/4 I

42314/Colombia_case_study.pdf

- Carvajal-Escobar, Y. (2004). El uso de funciones ortogonales empíricas y análisis de correlación canónica en el estudio de la variabilidad hidrometeorológica. Aplicación al Valle del Cauca - 
Colombia. Tesis doctoral, Universidad Politécnica de Valencia, España.

- _ (2010). Efectos de la variabilidad climática (VC) y el cambio climático en los recursos hídricos de Colombia. Revista Entre Ciencia e Ingeniería, 9, 33-61. preparados para enfrentar la variabilidad y el cambio climático? Revista Nacional de Investigación Memorias, 9(16), 105-119.

- . (2011b). Adaptación a la variabilidad climática y el cambio climático con enfasis en el contexto vallecaucano y colombiano. Trabajo para ascenso de escalafón a profesor titular, Universidad del Valle, Cali, Colombia.

- Carvajal-Escobar, Y. y Quintero, M. (2007). Que viva la montaña. Caso de Estudio Ginebra (Colombia). p. 6. Obtenido de: http://climadapta.univalle.edu.co/casos.htm

- CEPAL. (2010). Desastres y Desarrollo: El impacto en 2010 (Cifras preliminares) Unidad de Desastres. Unidad de Evaluación de Desastres. Boletín, 1, 1.

- Colombiassh.org. (Julio, 2011). Colombia inundaciones 2010-2011. Informe de situacion No. 36. Colombia ssh. Sala de Situación Humanitaria. Obtenido de: http://www.colombiassh.org/site/IMG/pdf/ Inundaciones_Colombia_Sit_Rep_36.pdf

- Collins, M. \& The CMIP Modelling Groups [BMRC (Australia), CCC (Canadá), CCSR/NIES (Japan), CERFACS (France), CSIRO (Australia), MPI (Germany), GFDL (USA), GISS (USA), IAP (China), INM (Russia), LMD (France), MRI (Japan), NCAR (USA), NRL (USA), Hadley Centre (UK) \& YNU (South Korea)]. (2005). El Niñoor La Niña-like climate change? Climate Dynamics, 24, 89-104.

- Debels, P.; Aldunce, P.; Bezanilla, A.; Carvajal-Escobar, Y.; Celis, A.; Martínez, M.; Néri, C., Quintero-Ángel, M. \& Szlafsztein, C. (2009). IUPA: a tool for the evaluation of the general use fulness of practices for adaptation to climate change and variability. Natural hazards, 50(2), 211-233.

- DANE -Departamento Administrativo Nacional de Estadística-. (2011). Boletín de prensa - Pobreza monetaria y multidimensional en Colombia. Colombia: DANE. Obtenido de: http://www.dane.gov.co/files/investigaciones/condiciones_vida I

pobreza/boletin_pobreza_2011.pdf

- Desinventar.org. (2011). Sistema de Inventario de Desastres de América Latina. C. Obtenido de: http://desinventar.org

- Duque, A. (2011). Colombia, octavo deforestador del mundo. UN Periódico. Universidad Nacional de Colombia. Obtenido el 29 de enero de 2012, de: http://www.unperiodico.unal.edu.co/dper/article/ colombia-octavo-deforestador-del-mundo-1.htm

- DNP -Departamento Nacional del Planeación-. (2011). Plan Nacional de Desarrollo 2010-2014. "Prosperidad para todos". Resumen ejecutivo. Bogotá: DNP. Obtenido de: https://www.dnp.gov.co/LinkClick.aspx? fileticket=4-J9V-FE2pl\%3D\&tabid=1238

- Gómez, H.. (2011). Construyendo resiliencia. Cambios institucionales para gestión del riesgo, variabilidad y cambio climatico. Foro por el Agua Semana.com. Bogotá. Departamento Nacional de Planeación. 

(2011a). Impactos y respuestas a la ola invernal 20102011. Retos para superar la vulnerabilidad a los fenómenos de origen natural en Colombia. Foro por el Agua. Bogotá: Departamento Nacional de Planeación.

- Guha-Sapir, D. (1997). Women in the front line: Women in developing countries should be key players in disaster preparedness and relief. París: UNESCO Courier. p. 27-29.

- Gutiérrez, S. (2013). Análisis de la influencia de El fenómeno ENOS "El Niño -Oscilación del Sur" en la oferta hídrica trimestral de la cuenca hidrográfica del río Dagua, Valle del Cauca. Tesis de pregrado, Universidad del Valle, Cali, Colombia. No publicada.

- GWA- Global Water Agency y WSP-Water and Sanitation Program. (2005). Taller Internacional "Construyendo una visión para la acción: Avances y desafíos de la transversalización del enfoque de género en la gestión integrada de recursos hídricos en América Latina". 9-19. Obtenido de: http://www-

s.worldbank.org/external/default/WDSContentServer/WDSP/IB/ 2005/10/03/000160016_20051003125815/Rendered/PDFI 330660lac1construyendo.pdf

- Hoyois, P. \& Guha Sapir, D. (2012). Measuring the Human and Economic Impact of Disasters. Informe. Centre for Research on Epidemiology of Disasters -CRED-. Obtenido de: http://www.bis.gov.uk/assets/foresight/docs/ reducing-risk-management/supporting-evidencel 12-1295-measuring-human-economic-impact-disasters.pdf

- IDEAM -Instituto de Hidrología, Meteorología y Estudios Ambientales-. (2001). El Medio Ambiente en Colombia. 2a ed. Bogotá, Colombia: IDEAM.

- _ (2004). Informe anual sobre el estado del medio ambiente y los recursos naturales renovables en Colombia. Bogotá, Colombia: IDEAM.

- _ (2010). Estudio Nacional del Agua. Bogotá, Colombia: IDEAM. Obtenido de: https://documentacion.ideam.gov.col.../021888.htm

- IGAC -Instituto Geográfico Agustín Codazzi-; IDEAM -Instituto de Hidrología, Meteorología y Estudios Ambientales- y DANE Departamento Administrativo Nacional de Estadística-. (2011). Reporte Consolidado de Áreas Afectadas por inundaciones, 20102011. Bogotá, Colombia. Obtenido de: https://www.siac.gov.co/contenido/contenido.aspx?catID=623\& conID=915

- Janssen, M.; Schoon, M.; Ke, W. \& Börner, K. (2006). Scholarly networks on resilience, vulnerability and adaptation within the human dimensions of global environmental change. Global Environmental Change, 16(3), 240-252.

- Lavell, A.; Mansilla, E. y Smith, D. (2004). La gestión local del riesgo: concepto y práctica. Managua: UNDP-CEPREDENAC.

- Magrin, G.; García, C.; Cruz, D.; Giménez, J.C.; Moreno, A.R.; Nagy, G.J.; Nobre, C. \& Villamizar, A. (2007). Latin America. Climate Change 2007: Impacts, Adaptation and Vulnerability. En: Contribution of Working Group II to the Fourth Assessment Report of the Intergovernmental Panel on Climate Change (pp. 581-615). Cambridge, UK: Cambridge University Press.

- MinHacienda. (2011). Consejo superior de política fiscal. Colombia: Minhacienda. 
http://www.minhacienda.gov.co/portal/page/portal/

HomeMinhacienda/politicafiscal/PlanFinanciero/2011/PLAN\%

2OFINANCIERA\%20-\%20003\%20-

\%202011\%20(Act\%20PF\%202011).pdf

- OMM. (2009). Tercera conferencia mundial sobre clima (CMC-3).

Obtenido de: http://www.wmo.int/wcc3/documents/WCC-

3_Statement_07-09-09_mods.pdf

- Oxfam. (2011). ¿Cómo evitar otro desastre? Lecciones de la ola invernal en Colombia. Informe 148. Obtenido de: http://www.oxfam.org/sites/www.oxfam.org/files/bp148_colomb ia-lecciones-aprendidas-final_spanish1104292.pdf

- Puertas, O. y Carvajal-Escobar, Y. (2008). Incidencia de El Niño Oscilación del Sur en la precipitación y la temperatura del aire en Colombia, Utilizando el Climate Explorer. Ingeniería \& Desarrollo, 23, 104-118.

- Sedano-Cruz, K. (2012). Manejo integrado del riesgo de inundaciones en Colombia. Tesis de Maestría, Departamento de Ingeniería Hidráulica y Medio Ambiente, Universidad Politécnica de Valencia, España. No publicada.

- Sedano-Cruz, K.; Carvajal, Y. \& Ávila, A. (2011). Variabilidad climática, cambio climático y gestión integrada del riesgo de inundaciones en Colombia. ¿Cómo nos afecta el cambio climático en Colombia? Revista semillas, 46(47), 47-53.

- Semana.com. (2011). La tragedia invernal en cifras. Citado en: El Reto, Revista ambiental on-line, 84, Mayo-junio de 2011, p. 23. Obtenido el 30 de abril de 2011, de: http://xa.yimg.com/kq/groups/10531607/891728550/name/Edici \%C3\%B3n+84+Mayo-+Junio+2011.pdf

- Shrader, E. \& Delaney, P. (2000). Gender and post-disaster reconstruction: The case of Hurricane Mitch in Honduras and Nicaragua. The World Bank Report. Obtenido de: $\mathrm{ftp}: / / f t p . s h e f . a c . u k / p u b / u n i / a c a d e m i c / A-$

Clar1mb/WB_GenderAndPost

DisasterReconstruction.doc

- SNPAD. (2011). Consolidado de Atención de Emergencias; Sistema Nacional para la Prevención y atención de Desastres. Obtenido de: http://www.sigpad.gov.co/sigpad/emergencias_detalle.aspx?id $\mathrm{n}=41$

- Swiss Re. (2011). Sigma No 1/2011. Zusich: Compañía Suiza de Reaseguros S.A. Economic Research \& Consulting.

- Szlafsztein, C.; Debels, P.; Aldunce, P.; Neri, C.; Carvajal-Escobar, Y.; Celis, A.; Bezanilla, A.; Quintero, M. y Martínez, D. (2007). Hacia la evaluación de prácticas de adaptación ante la variabilidad y cambio climático en América Latina y el Caribe . Brasil. p. 109.

- Tompkins E. \& Adger, W. (2005). Defining response capacity to enhance climate change policy. Environ Sci Policy, 8(6), 562-571. Citado en: Debels et al. (2009). IUPA: a tool for the evaluation of the general Usefulness of Practices for Adaptation to climate change and variability.

- UN/DESA. (2005). Population challenges and development goals. Departament of economic and social affairs. Obtenido de: http://www.un.org/esa/population/publications/pop_challenges/ Population_Challenges.pdf

- UNGRD -Unidad Nacional para la Gestión del Riesgo de Desastres-. (2011). Reporte oficial de afectación y respuesta II temporada de Iluvias. Colombia: UNGRD. 
- Vincent, K. (2007). Uncertainty in adaptive capacity and the importance of scale. Global Environ Change, 17, 12-24.

- Wolter, K. \& Timlin, M. (1993). Monitoring ENSO in COADS with a seasonally adjusted principal component index. Proc. of the 17th Climate Diagnostics Workshop. Norman, OK, NOAA/NMC/CAC, NSSL, Oklahoma Clim. Survey, CIMMS and the School of Meteor., Univ. of Oklahoma. p. 52-57. Obtenido de: http://www.esrl.noaa.gov/psd/enso/mei/WT1.pdf

- . (1998). Measuring the strength of ENSO events - how does 1997/98 rank? Weather, 53, 315-324.

- _ (2011). Comportamiento El Niño / Southern Oscillation behaviour since 1871 as diagnosed in an extended multivariate ENSO index. Intl. J. Climatology, 31, 14p. In press. Obtenido de: http://www.esrl.noaa.gov/psd/enso/mei/\#data

- Yonder, A., Akcar, S., \& Gopalan, P. (2005). Women's participation in disaster relief and recovery. Seeds 22, Population Council. New York.

1. Candidata a Ph.D en Ingeniería del Agua y Medio Ambiente. M.Sc de Ingeniería Hidráulica y Medio Ambiente, Universidad Politécnica de Valencia. Ingeniera Sanitaria, Universidad del Valle. Beneficiaria Beca Francisco José de Caldas de Colciencias. Colaboradora del Grupo IREHISA, Universidad del Valle, Colombia. Grupo de Investigación en Ingeniería de los Recursos Hídricos y Suelos -IREHISA-, Universidad del Valle, Colombia. rutsecru@posgrado.upv.es

Correspondencia de autor: Ruth Karime Sedano Cruz, Rua dos Jacarandás 300, Condominio Saint Martin. BI. 5 - Apto 1302, C.E.P. 22776-050, Barra da Tijuca, Rio de Janeiro, Brasil. Tel: +55 (21) 98807558. rutsecru@posgrado.upv.es

2. Ph.D en Hidráulica y Medio Ambiente. Diploma de Estudios Avanzados en Hidráulica y Medio Ambiente. M.Sc en Suelos y Aguas, Universidad Nacional de Colombia. Ingeniero Agrícola, Universidad del Valle Universidad Nacional de Colombia. Director Grupo IREHISA, Profesor Escuela de Ingeniería de los Recursos Naturales y del Ambiente EIDENAR-, Universidad del Valle, Colombia. Grupo de Investigación en Ingeniería de los Recursos Hídricos y Suelos -IREHISA-, Universidad del Valle, Colombia. yesid.carvajal@correo.univalle.edu.co

3. Ing. Agrícola, Universidad del Valle. Joven Investigador de Colciencias. Colaborador del Grupo IREHISA, Universidad del Valle, Colombia. Grupo de Investigación en Ingeniería de los Recursos Hídricos y Suelos IREHISA-, Universidad del Valle, Colombia. alvaro.avila@correounivalle.edu.co

4. Carrera 100 No. 13-00. Ciudad universitaria Meléndez. Edificio 344, Oficina 2007, Cali, Colombia. Tel/Fax: +57 (2) 3396097 ext. 107/22. http://irehisa.univalle.edu.co/index.html 
Para citar este artículo: Sedano-Cruz, K., Carvajal-Escobar, Y. \& Ávila, Á. J. (2013). Análisis de aspectos que incrementan el riesgo de inundaciones en Colombia. Revista Luna Azul, 37, 219238.

Recuperado

de

http://lunazul.ucaldas.edu.co/index.php?option=content\&task=view\&id=853 